\title{
Inhomogeneous melting in anisotropically confined two-dimensional clusters
}

\author{
S. W. S. Apolinario, ${ }^{*}$ B. Partoens, ${ }^{\dagger}$ and F. M. Peeters ${ }^{\ddagger}$ \\ Departement Fysica, Universiteit Antwerpen, Groenenborgerlaan 171, B-2020 Antwerpen, Belgium \\ (Received 18 January 2006; revised manuscript received 14 July 2006; published 11 September 2006)
}

\begin{abstract}
Molecular dynamic simulations are performed to investigate the melting process of two-dimensional clusters of classical charged particles trapped in an anisotropic parabolic potential. The confined particles interact through a repulsive potential. We find that the eccentricity of the confinement potential strongly affects the melting pattern of such clusters. Increasing the eccentricity of the confinement potential drives the system through three different melting regimes. Inhomogeneous melting is the typical melting process for anisotropically confined clusters and its appearance in small systems occurs in a distinct form called here internal intershell melting. The latter involves only particles in the center of the cluster while particles on the far left and right of the cluster are still ordered having a much higher melting temperature. Using the Lindemann's criterion the melting temperatures are determined as a function of the different parameters. The internal intershell melting process is found for both long-range (i.e., logarithmic) and short-range (i.e., screened Coulomb) interparticle interaction. Decreasing the range of the interparticle interaction increases the eccentricity of the confinement potential for which internal intershell melting can occur.
\end{abstract}

DOI: 10.1103/PhysRevE.74.031107

PACS number(s): 64.60.-i, 63.22.+m

\section{INTRODUCTION}

During the past several years many efforts have been directed to understanding static and dynamical properties of mesoscopic devices in which the interacting particles are laterally confined. Typical experimental realizations of such two-dimensional (2D) systems include electrons on the surface of liquid helium [1], electrons in quantum dots [2], colloidal particles [3], vortices in mesoscopic-shaped superconductors $[4,5]$, confined plasma crystals [6], and a confined system of metallic balls $[7,8]$.

The interparticle interaction ranges from a Coulomb potential, logarithmic interaction, screened Coulomb to a dipole interaction. Vortices in a film of liquid helium interact through a logarithmic potential [9], as do a low concentration of vortices in a type II superconducting 2D film [10]. The logarithmic interaction between vortices was used to study the stable vortex configurations in a disk-shaped superconductor $[11,12]$. Recently, it was shown $[7,8]$ that the configurations of charged metallic balls placed on the bottom of a plane capacitor could be described by a logarithmic interparticle potential.

Historically, Wigner [13] predicted in 1934 that electrons in a three-dimensional Fermi system at low temperature and density undergo an electron-liquid to electron-crystal phase transition. Such an electron-crystal is called a Wigner crystal. To the best of our knowledge no clear observation of such an electron crystal in three dimensions (3D) has been made up to now. In 1971 Crandall and Williams [14] noted that an analogous phase transition should occur in a classical 2D electron system at sufficiently high density which was first observed by Grimes and Adams [1] in an electron gas confined above the surface of liquid helium. For this kind of

\footnotetext{
*Electronic address: sergio.apolinario@ua.ac.be

†Electronic address: bart.partoens@ua.ac.be

*Electronic address: francois.peeters@ua.ac.be
}

system with a circular symmetric interaction potential and in the absence of a corrugated surface, the solid phase with the lowest energy has a triangular lattice structure independent of the electron density [15]. However, a 2D confined system with a finite number of ions or electrons does not crystallize in a triangular lattice (Wigner crystal), but the particles are arranged in a shell structure as was predicted in Ref. [16]. For a large finite cluster the structure is due to the competition between the ordering into a triangular lattice symmetry and the circular symmetry imposed by the confining potential.

Reference [8] investigated experimentally the effect of the anisotropic confinement on the ground and metastable states of metallic balls trapped by an anisotropic confinement potential. It was shown that the asymmetry of the confinement potential induces various rearrangements of the particles, according to their number and the asymmetry of the potential. They also investigated metastable configurations of the system and well-defined triangular structures, which are induced by the eccentricity of the confinement potential. This experimental work [8], inspired us [17] to investigate the effect of the symmetry of the confinement on the configurations of the particles. We considered a model of classical charged particles interacting through a logarithmic interaction potential as suggested by the experiment. We reobtained theoretically all configurations observed in Ref. [8]. Many transitions as a function of the eccentricity of the confinement potential were found and could be classified as first and second order, which, respectively, exhibit a discontinuity in the first and second derivative of the energy. Also we showed that these transitions affect the eigenmode spectrum of the system. In an earlier work [18], Coulomb interacting particles confined by an elliptic potential were investigated theoretically, but at that time no experimental results were available.

Phase transitions in two-dimensional large crystals have mainly been described by changes in the asymptotic behavior of spatial correlation functions. For instance, the Kosterlitz-Thouless-Halperin-Nelson-Young (KTHNY) theory predicts a two-step melting scenario according to 
which the liquid phase is reached when bond-orientational correlations become short range [19-24]. This scenario differs considerably from what is generally predicted to occur with small 2D clusters in an isotropic trap when the temperature is raised. For the latter system, melting is theoretically predicted to occur via a two-step process [16,25-27]. At very low temperature, each particle is thermally excited in its local potential. Upon increasing the temperature, angular rotation becomes possible where orientational order between adjacent shells is lost, which is called angular melting. Consecutively, for higher temperatures an intrashell melting occurs and at the same time radial diffusion between shells takes place.

In this paper we investigate how the different melting processes are influenced by the geometry of the confinement potential. Therefore, we study the melting process in $2 \mathrm{D}$ model systems of $N$ equally charged particles trapped by an elliptic confinement potential and interacting through a repulsive interparticle potential. We focus on the effect of the eccentricity of the confinement potential on the melting process of the system. We found that the eccentricity of the confinement potential induced different melting processes; some of them we found are different from the circular confined system. The dependence of these melting processes on the range of the interparticle potential is studied by considering a logarithmic and screened Coulomb interparticle potential. For sufficient large asymmetry of the confinement we found a temperature-induced breakup of the sample in different regions, which have a different melting temperature.

The paper is organized as follows. In Sec. II our model system and the methodology used to find stable configurations is given. In Sec. III we deal with the system of classical particles interacting through a logarithmic interparticle potential. First, we investigate qualitatively the melting processes in relatively small clusters of particles $N=6,12,13$ and 30 . These examples concern clusters with a distinct number of shells and provide an understanding of the main melting processes occurring in anisotropic clusters. Next, the effect on inhomogeneous melting by the size of the cluster is investigated and melting temperatures are obtained. In Sec. IV we investigate how our results depend on the type of interaction considering a cluster with $N=13$ particles for a screened Coulomb interparticle potential. Finally, in Sec. V we present our conclusions.

\section{MODEL AND NUMERICAL APPROACH}

We study a 2D model system of $N$ equally charged particles in an elliptic confinement potential and interacting through a repulsive potential. The potential energy of the system is given by

$$
E=\sum_{i=1}^{N} \frac{1}{2} m\left(\omega_{0 x}^{2} x_{i}^{2}+\omega_{0 y}^{2} y_{i}^{2}\right)+\sum_{i>j}^{N} V\left(\left|\mathbf{r}_{i}-\mathbf{r}_{j}\right|\right),
$$

where $m$ is the mass of the particle, $\mathbf{r}_{i}=\left(x_{i}, y_{i}\right)$ is the vector position of the $i$ th particle, $V(\mathbf{r})$ is the repulsive interparticle interaction, and $\omega_{0 x}$ and $\omega_{0 y}$ are, respectively, the confinement frequencies in the $x$ and $y$ directions. These fre- quencies are related by the expression $w_{0 x}=\sqrt{\alpha} \omega_{0 y}$, where $\alpha$ is the eccentricity of the elliptic confinement. In the present study we consider a logarithmic interparticle interaction $V(\vec{r})=-\beta \ln |\vec{r}|$ and a screened Coulomb potential $V(\vec{r})=\left(q^{2} / \epsilon\right) \exp (-|\vec{r}| / \lambda) /|\vec{r}|$.

We can write the potential energy (1) in dimensionless form if we express the coordinates, energy, temperature, and time, respectively, in the following units: $r_{0}=\left(2 \beta / m \omega_{0 y}^{2}\right)^{1 / 2}$, $E_{0}=\beta, T_{0}=\beta k_{B}^{-1}$, where $k_{B}$ is the Boltzmann constant and $t_{0}=\sqrt{2} / \omega_{0 y}$ in the case of a logarithmic confinement potential. The dimensionless potential energy is

$$
E=\sum_{i=1}^{N}\left(\alpha x_{i}^{2}+y_{i}^{2}\right)-\sum_{i>j}^{N} \ln \left|\mathbf{r}_{i}-\mathbf{r}_{j}\right|
$$

For a screened Coulomb potential this becomes

$$
E=\sum_{i=1}^{N}\left(\alpha x_{i}^{2}+y_{i}^{2}\right)+\sum_{j>i}^{N} \frac{\exp \left(-\kappa\left|\mathbf{r}_{i}-\mathbf{r}_{j}\right|\right)}{\left|\mathbf{r}_{i}-\mathbf{r}_{j}\right|}
$$

with, respectively, for the units of length, energy, temperature, and time $r_{0}=\left(q^{2} / \epsilon \gamma\right)^{1 / 3}$ where $\gamma=m \omega_{0 y}^{2} / 2, E_{0}=\gamma r_{0}^{2}$, $T_{0}=E_{0} k_{B}^{-1}$, and $t_{0}=\sqrt{2} / \omega_{0 y}$. The dimensionless inverse screening length, $\kappa=r_{0} / \lambda$, is a measure of the range of the interparticle interaction potential. All the results will be given in dimensionless units.

The stable configuration is a local or global minimum of the potential energy, which is only a function of the number of charged particles $N$ and the eccentricity $\alpha$ (and $\kappa$ in case of a screened Coulomb interaction). Our numerical method to obtain the stable-state configuration is based on the Monte Carlo simulation technique supplemented with the Newton method in order to increase the accuracy of the found energy value [26]. By starting from many different random initial configurations we believe that we were able to find all the possible stable (i.e., ground state and metastable) configurations. These configurations were discussed in our previous paper [17].

To study the dynamical properties of a small cluster at a specific temperature we implement the molecular dynamic (MD) simulation in three different stages. (1) We use a variant of the velocity Verlet algorithm [28], which rescales the velocity of the particles to bring the sample to a desired temperature. The rescaling of the velocities in such an algorithm implies that we are no longer following Newton's equations, and no data should be collected in this stage. (2) The last configuration obtained in the previous step serves as an initial condition in a subsequent molecular dynamics (MD) simulation using the velocity Verlet algorithm, which brings the system to a statistical equilibrium state. (3) The last configuration in the second step is integrated in time using the velocity Verlet algorithm while data are collected. A typical measurement done during this latter stage is the calculation of the averaged displacement of the particles from its equilibrium position [16]. 


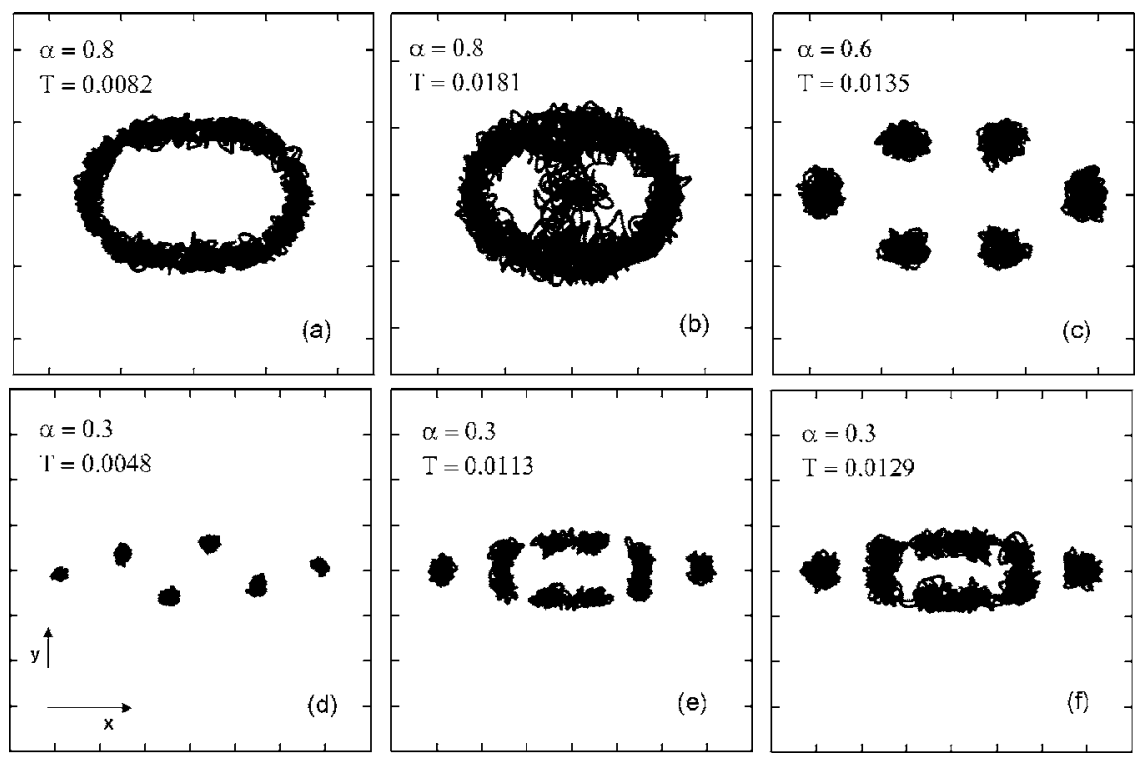

FIG. 1. Particle trajectories for a system with $N=6$ particles, different values of temperature, and three eccentricity confinement values $\alpha=0.8,0.6$, and 0.3 . The scale is different in each figure, but the distance between the ticks is always one length unit.

\section{MELTING TRANSITION FOR SYSTEMS WITH LOGARITHMIC INTERPARTICLE POTENTIAL}

In this section we demonstrate how the eccentricity of the confinement potential affects the melting in systems with a logarithmic interparticle potential. Therefore, we consider systems ranging from $N=6$ up to $N=60$ particles. These examples involve clusters with a distinct number of shells. In the following we show detailed results for four different clusters, which contain typical melting processes we observed: a system with one shell that encloses no particles ( $N=6$ particles), systems with one shell but enclosing particles ( $N=12$ and 13 particles), and a system with two shells $(N=30$ particles). Finally, we investigate larger clusters with $N=40,50$, and 60 particles. The melting phenomenon for clusters with a different number of particles can be deduced from these results. To acquire insight into the diverse melting processes and their dependence on the eccentricity parameter we show typical particle trajectories performed during a relatively small time interval in a MD simulation. Next, different melting temperatures are obtained quantitatively as a function of $\alpha$ by using Lindemann's criterion. We will see that a weak anisotropic system exhibits the same melting processes as in a system with circular confinement potential, i.e., angular melting followed by a radial melting. We show how the melting temperature of these processes changes with the eccentricity of the confinement potential. For small and strong anisotropic systems $(\alpha<0.5)$, an inhomogeneous melting process appears untypical for circular systems, which we call internal intershell melting.

The small cluster with $N=6$ particles has one shell configuration $(0,6)$ for $\alpha \neq 1$ and $(1,5)$ when $\alpha=1$ and we will investigate how the eccentricity of the confinement potential influences the melting of such a system. Considering this latter configuration as a starting position in a MD simulation, we obtained the trajectory patterns shown in Fig. 1 for different values of the eccentricity and temperature. These tra- jectories were obtained by collecting the position of the particles during a time interval of $\Delta t=500$. For small anisotropy, for example, $\alpha=0.8$, the system of $N=6$ particles exhibits the well-known two-step melting process like for an isotropic system. The first melting process, called angular melting, occurs at the temperature $T=0.0082$ [Fig. 1(a)], while radial melting, where particles can jump between stable configurations $(0,6)$ and $(1,5)$, occurs for a temperature of $T=0.0181$ [Fig. 1(b)]. The first consequence of the anisotropic confinement is that decreasing $\alpha$ leads to an increase of the angular melting temperature. For example, for a relatively high temperature $T=0.0135$ and $\alpha=0.6$ [Fig. 1(c)] the particles are still oscillating around their equilibrium position and no angular melting is observed. For an extremely anisotropic system $\alpha=0.3$ at low temperature, for example, $T=0.0048$ [Fig. 1(d)], particles perform a simple oscillation around their equilibrium position. However, for increasing temperature, particles are first [Fig. 1(e)] able to occupy both degenerated ground state configurations and consecutively, in a second step at higher temperature $T=0.0129$, the four central particles lose their angular orientational order in a dynamic process that we will call internal intrashell melting. In contrast, the two particles located in the most extremum position in the cluster have an oscillatory motion around their equilibrium position. Also, we can verify from Figs. 1(e) and 1(f) that increasing the eccentricity of the confinement potential in a system with $N=6$ particles induces regions in the cluster having a different melting temperature. In order to be able to describe these melting processes quantitatively, we distinguish two regions in Fig. 1(d). Region 1 holding two particles is placed at the left and right extremum of the cluster while region 2 , which is the center of the cluster, contains four particles.

In previous work [16] the melting temperature was determined quantitatively by calculating the radial and angular averaged displacement. However, for anisotropic systems with elliptic confinement it is more convenient to calculate the $x$ averaged displacement defined as 


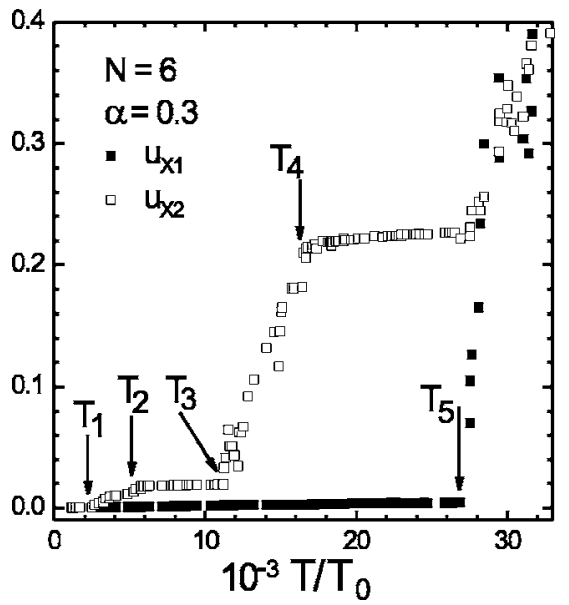

FIG. 2. The $x$-averaged displacement for regions 1 [full squares, left and right particles in Fig. 1(d)] and 2 [empty squares, center four particles in Fig. 1(d)].

$$
u_{x \gamma}^{2}=\frac{1}{N_{\gamma}} \sum_{i=1}^{N_{\gamma}}\left(\left\langle x_{\gamma i}^{2}\right\rangle-\left\langle x_{\gamma i}\right\rangle^{2}\right) / a^{2},
$$

where $\gamma=1$ and 2 indicates the region number, $N_{\gamma}$ is the number of particles in region $\gamma, x_{\gamma i}$ is the $x$ coordinate of the $i$ th particle of group $\gamma$, and $a$ is the average distance between the particles. The calculation of the $x$-averaged displacement as function of temperature allowed us to determine the different melting processes quantitatively.

The reason to use the $x$-averaged displacement is that for high anisotropic confinement the system presents regions along the $x$ direction with distinct dynamical properties. We define the melting temperature as the temperature at which the $x$-averaged displacement changes its linear temperature dependence into a more rapid increase.

Through a MD simulation where we collected data during a time interval of $\Delta t=10^{5}$ we obtained the temperature dependence of the $x$-averaged displacement (Fig. 2) for the particles in regions 1 (full squares) and 2 (empty squares) for the system with $N=6$ particles and $\alpha=0.3$. We can clearly see three melting regions and two plateaus. The first melting process, where the system becomes able to oscillate between both degenerated ground-state configurations starts at the temperature $T_{1}=2.46 \times 10^{-3}$. For this latter value of the temperature the $x$ averaged displacement of group two $\left(u_{x 2}\right)$ changes its initial slope present at low temperatures. A first plateau which presents a dynamical equilibrium is found for the temperature range from $T_{2}=5.73 \times 10^{-3}$ to $T_{3}=11.24$ $\times 10^{-3}$. For this temperature interval, $u_{x 2}$ shows a very small linear increase. The internal angular melting starts to occur at $T_{3}=11.24 \times 10^{-3}$ when $u_{x 2}$ shows a sudden increase. A second plateau for $u_{x 2}$ occurs for the range of temperatures varying from $T_{4}=17.22 \times 10^{-3}$ to $T_{5}=26.52 \times 10^{-3}$ corresponding to a complete internal angular melting. This melting process is followed by a full melting, which happens at temperature $T_{5}=26.52 \times 10^{-3}$ where both $x$ averaged displacement of groups $1\left(u_{x 1}\right)$ and $2\left(u_{x 2}\right)$ diverges.

Let us now look at a larger cluster. Reference [8] showed that large anisotropic confined systems have a ground-state configuration with a regular arrangement for which the packing is close to a hexagonal symmetry. This regular arrangement is induced by the anisotropic confinement. The groundstate configuration for $N=13$ particles and $\alpha=0.4$ also exhibits a regular structure. We investigate the melting process as a function of the eccentricity of the confinement potential. The melting process for the system with $N=13$ particles strongly depends on the eccentricity and, in particular, for $\alpha=0.4$ the system exhibits a distinct melting pattern. We present in Fig. 3 typical trajectory patterns. For an anisotropic confinement of $\alpha=0.7$ a system with $N=13$ particles (the first row in Fig. 3) exhibits intershell melting at a temperature of $T=0.00244$ [Fig. 3(b)]. Radial melting occurs at $T=0.00956$ [Fig. 3(d)] where the central particle can jump to the outer shell and a particle in the shell can jump to the central area. For a larger temperature $(T=0.01597)$ the two neighbor particles of the central particle are also able to jump to the outer shell and therefore also contribute to the radial melting process [Fig. 3(e)]. With increasing anisotropy the intershell melting temperature also increases. For a more anisotropic system with $\alpha=0.6$ (the second row in Fig. 3) we did not find intershell melting even for $T=0.00603$ [Fig. $3(\mathrm{~g})]$. For this system intershell and radial melting is found at $T=0.0091$ and $T=0.017$, respectively, as shown in Figs. 3(h) and 3(i). If we increase the eccentricity further, one can see from the third row of Fig. 3 for $\alpha=0.45$ that intershell melting no longer occurs. Increasing the temperature leads immediately to a full radial melting. Typical trajectories showing the radial melting are displayed in Fig. 3(o) and 3(p) for the temperatures $T=0.00956$ and $T=0.01243$, respectively. A traditional intershell melting, like the one present in a circular confined system, also does not appear for the system with eccentricity $\alpha=0.4$ (the fourth row in Fig. 3). However, a different type of melting process was found, a new internal shell is formed, which starts to melt first with increasing temperature, while the particles located at the corners only perform small oscillations [see Fig. 3(t) for $T=0.0099$ ]. We denote this type of melting as internal intershell melting and is a consequence of a temperature-induced breakup of the system into different regions. For $T=0.01336$ radial melting is found [Fig. 3(u)].

The particle trajectories for increasing temperatures in the last row of Fig. 3 show that the dynamical behavior of a cluster with $N=13$ particles and $\alpha=0.4$ strongly varies along the $x$ direction of the cluster. We can distinguish two regions with different dynamic properties in Fig. 3(q). Region 1 placed in the extreme left side of the cluster has three particles forming a triangular arrangement; and region 2 , which is the center of the cluster, has seven particles with one at the origin of the coordinate system and the six others are placed in the corner of a hexagon around the central particle. The melting temperature for the system with 13 particles and eccentricity $\alpha=0.4$ and 0.6 was determined by calculating the $x$-averaged displacement. Besides the $x$-averaged displacement of regions 1 and 2 we defined two new expressions: the $x$-averaged displacement of particles of the outer shell, $u_{x s}$, and the $x$-averaged displacement for the particles inside the shell, $u_{x i}$. With these two latter definitions we can also characterize the critical temperature of the intershell melting process. Figure 4(a) shows the dependence of the 

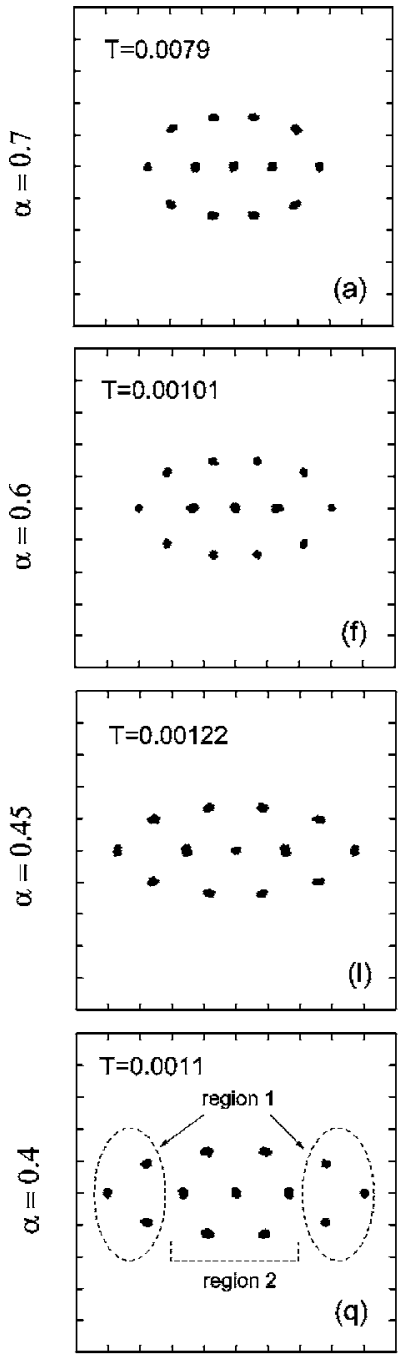
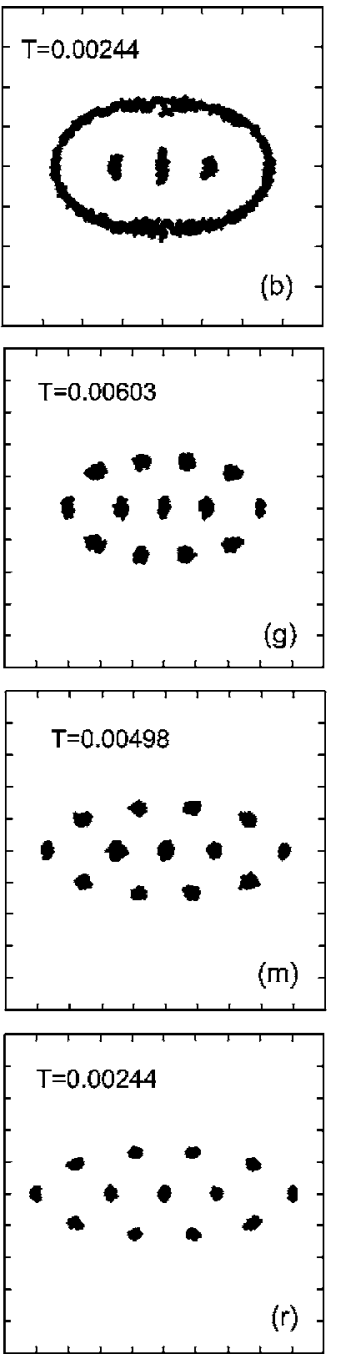

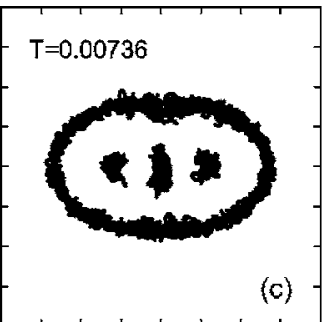

(c)
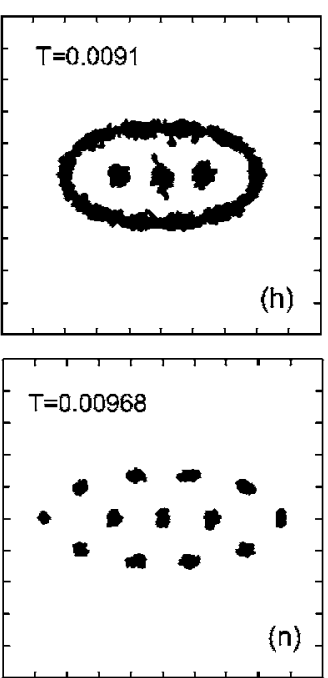

(n)

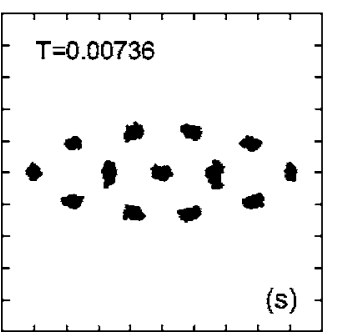

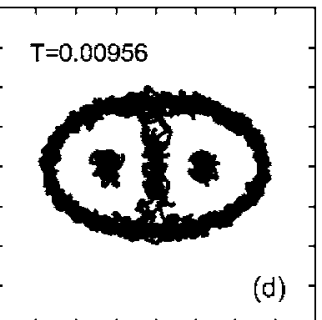
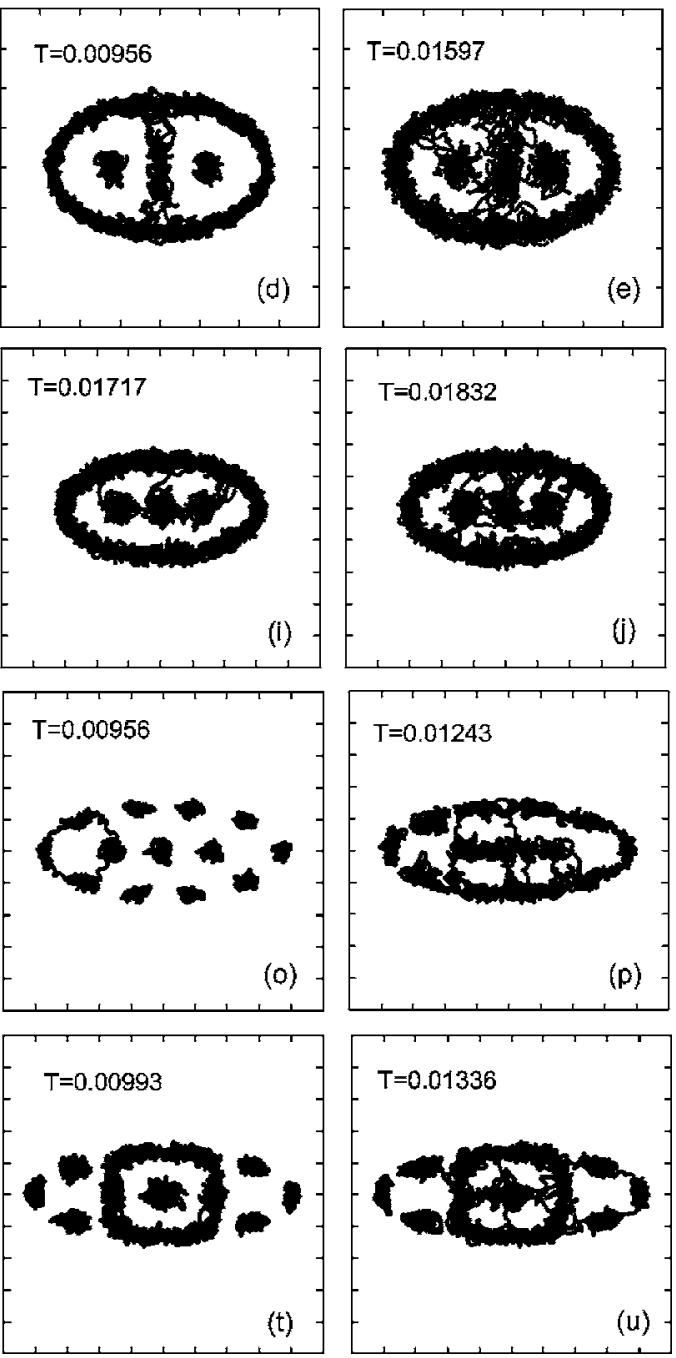

FIG. 3. Particle trajectories obtained during a time interval $\Delta t=500$ for a system with $N=13$ particles. Each row corresponds to the same value of $\alpha$ and along the column from left to right the temperature increases. The distance between the ticks is one length unit.

$x$-averaged displacement as a function of temperature for groups of particles in regions1 (full squares) and 2 (empty squares) for a system with $N=13$ particles and eccentricity $\alpha=0.4$. For increasing temperature, the latter system first exhibits an internal intershell melting at temperature $T_{i i m}=2.65 \times 10^{-3}$ and second a radial melting process at temperature $T_{r}=5.42 \times 10^{-3}$. For a less anisotropic confinement potential, $\alpha=0.7$, the system with $N=13$ particles exhibits an intershell melting process at temperature $T_{i m}=0.13 \times 10^{-3}$ [Fig. 4(b)], where the $x$-averaged displacement of particles in the shell diverges. A full melting process is reached at $T_{r}=2.63 \times 10^{-3}$ when $u_{x i}$ starts to diverge.

It is well known that the so-called magic-number clusters in systems with circular confinement have a higher intershell melting temperature, which is a consequence of a commensurability between particles in different shells. We also investigate the influence of the symmetry on the internal intershell melting process. Differently from the configuration for $N$ $=13$ particles and $\alpha=0.4$, the configuration for $N=12$ particles and $\alpha=0.4$ is not symmetric with respect to a reflection around $x=0$. The trajectories for the system with $N=12$ particles and $\alpha=0.4$ at the average temperature $T=0.0004$ and
$T=0.0021$ are shown, respectively, in Figs. 5(a) and 5(b). The dynamical behavior of the system is not homogeneous along the $x$ direction. While particles in the center of the cluster exhibit internal intershell melting [Fig. 5(b)], the particles at the corners of the cluster oscillate around their equilibrium position. For a quantitative characterization of the different melting processes present in the system we defined two different regions in the cluster shown in Fig. 5(a). Region 1 placed on the extreme left side of the cluster has three particles forming a triangular arrangement, while region 2 is the center of the cluster and contains 6 particles with five particles forming a pentagon, which encloses the sixth particle. The dependency of the $x$-averaged displacement on temperature for the particles in regions 1 (full squares) and 2 (empty squares) for the system with $N=12$ particles and eccentricity $\alpha=0.4$ is shown in Fig. 4(c). The internal angular and radial melting temperatures for this system are, respectively, $T_{i i m}=1.12 \times 10^{-3}$ and $T_{r}=5.66 \times 10^{-3}$ as indicated in Fig. 4(c). At the same eccentricity of $\alpha=0.4$ the value of the internal intershell melting temperature is higher for the system with $N=13$ particles $\left(T_{\text {iim }}=2.65 \times 10^{-3}\right)$ than for the system with $N=12$ particles showing that the asymmetry of the 


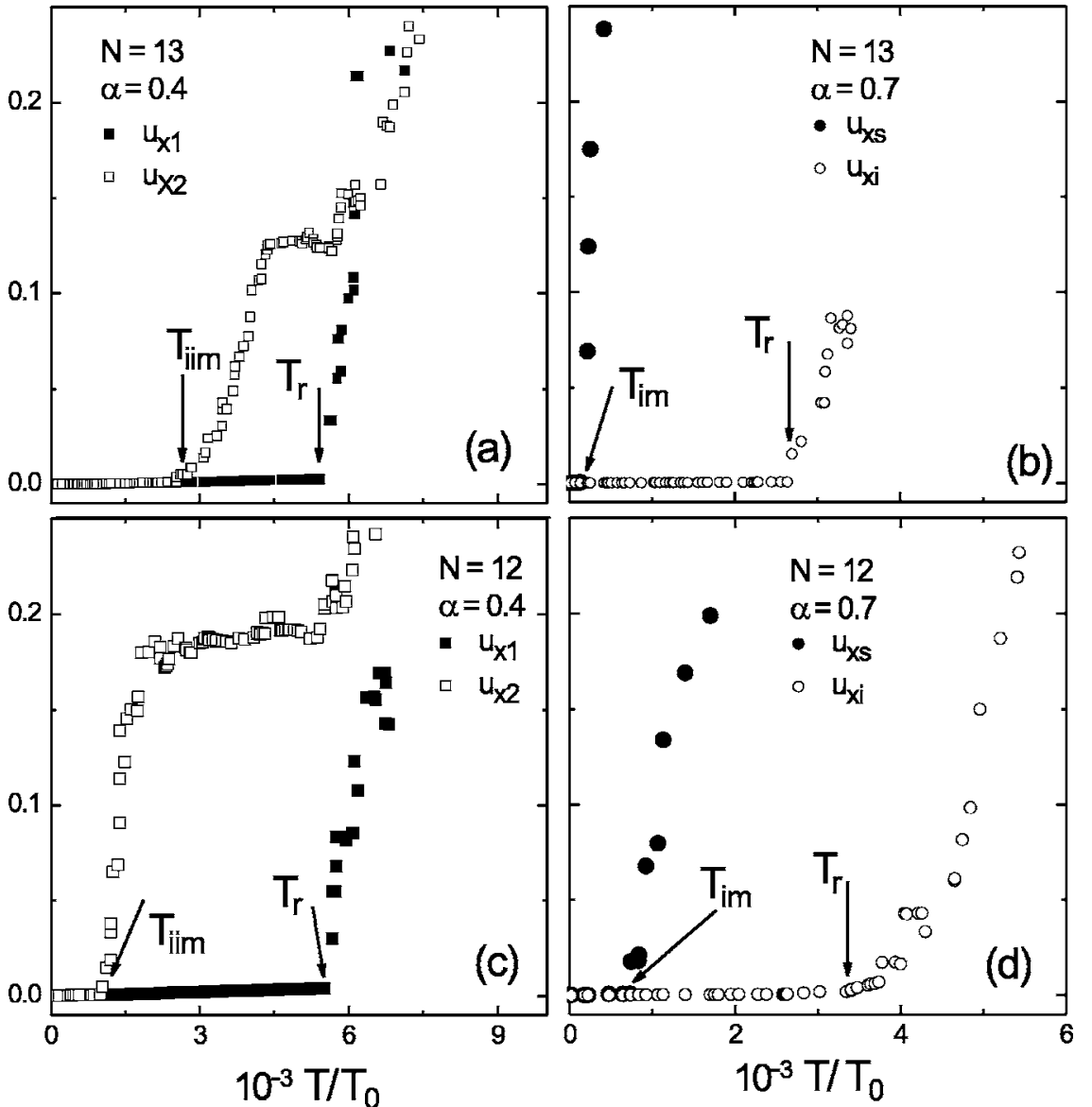

FIG. 4. The temperature dependence of the $x$-averaged displacement [Eq. (4)] for the groups of particles in regions 1 and 2 [see Figs. 3(q) and 5(a)] of the systems with $N=12$ and 13 particles and two eccentricity values $\alpha=0.4$ and $\alpha=0.7$. cluster decreases the stability of the cluster. In order to define the intershell melting temperature for the system with $N$ $=12$, we also defined the $x$-averaged displacement for the particles in the outer shell, $u_{x s}$, and for the particles inside the shell, $u_{x i}$. The intershell melting temperature for the system with $N=12$ and $\alpha=0.7$ is $T_{\text {im }}=0.74 \times 10^{-3}$ [Fig. 4(d)], which is followed by a radial melting at $T_{r}=3.40 \times 10^{-3}$. We also confirmed that the intershell melting temperature increases with increasing anisotropy of the confinement potential. The critical temperature for intershell melting for the system with $N=13$ particles and eccentricity $\alpha=0.7,0.65$, and 0.6 are, respectively, $T_{i m}=0.32 \times 10^{-3}, 0.85 \times 10^{-3}$, and $2.45 \times 10^{-3}$, while for the system with $N=12$ and eccentricity $\alpha=0.7$,

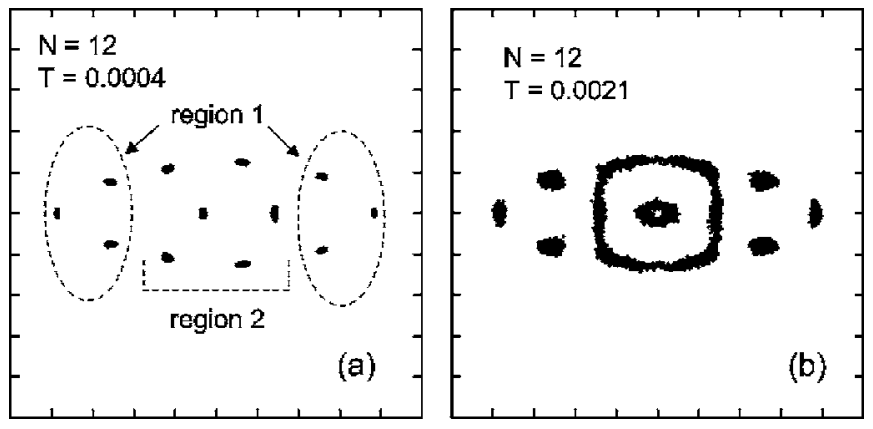

FIG. 5. Particle trajectories obtained for a time interval $\Delta t=500$ for a system with $N=12$ particles and anisotropy confinement of $\alpha=0.4$. The distance between the ticks is one length unit.
0.65 , and 0.6 , the critical temperatures are substantially larger and are, respectively, $T_{i m}=0.74 \times 10^{-3}, 1.57 \times 10^{-3}$, and $2.7 \times 10^{-3}$.

The angular melting can qualitatively be understood by considering the momentum transfer (i.e., collisions) between nearest neighbors in a shell. At low temperature, the intershell rotational mode dominates the dynamics of the system. Angular melting can occur when a critical kinetic-energy value is reached to overcome the barrier and particles move predominantly in the same clockwise or counterclockwise direction. However, when a strong enough anisotropic confinement is applied, the shell structure is stretched along the weakest confinement direction and a collision between two particles does not cause the particle at the corner to make a turn. This stops the original angular melting and the oscillations of the particles at the corners become relatively smaller than those of the particles in the middle of the cluster. Under these circumstances, radial melting becomes the first melting process for increasing temperature. For higher anisotropy, the shell structure is restored in the center of the cluster where angular melting becomes possible again.

In order to see if the above physics is also present at larger systems, we took $N=30$ particles for which the ground-state configuration has two shells. This will tell us how the number of shells influences the melting processes found previously for systems with one shell. We found that the melting for this latter system is very similar to the melting process for a system with $N=13$ particles. 

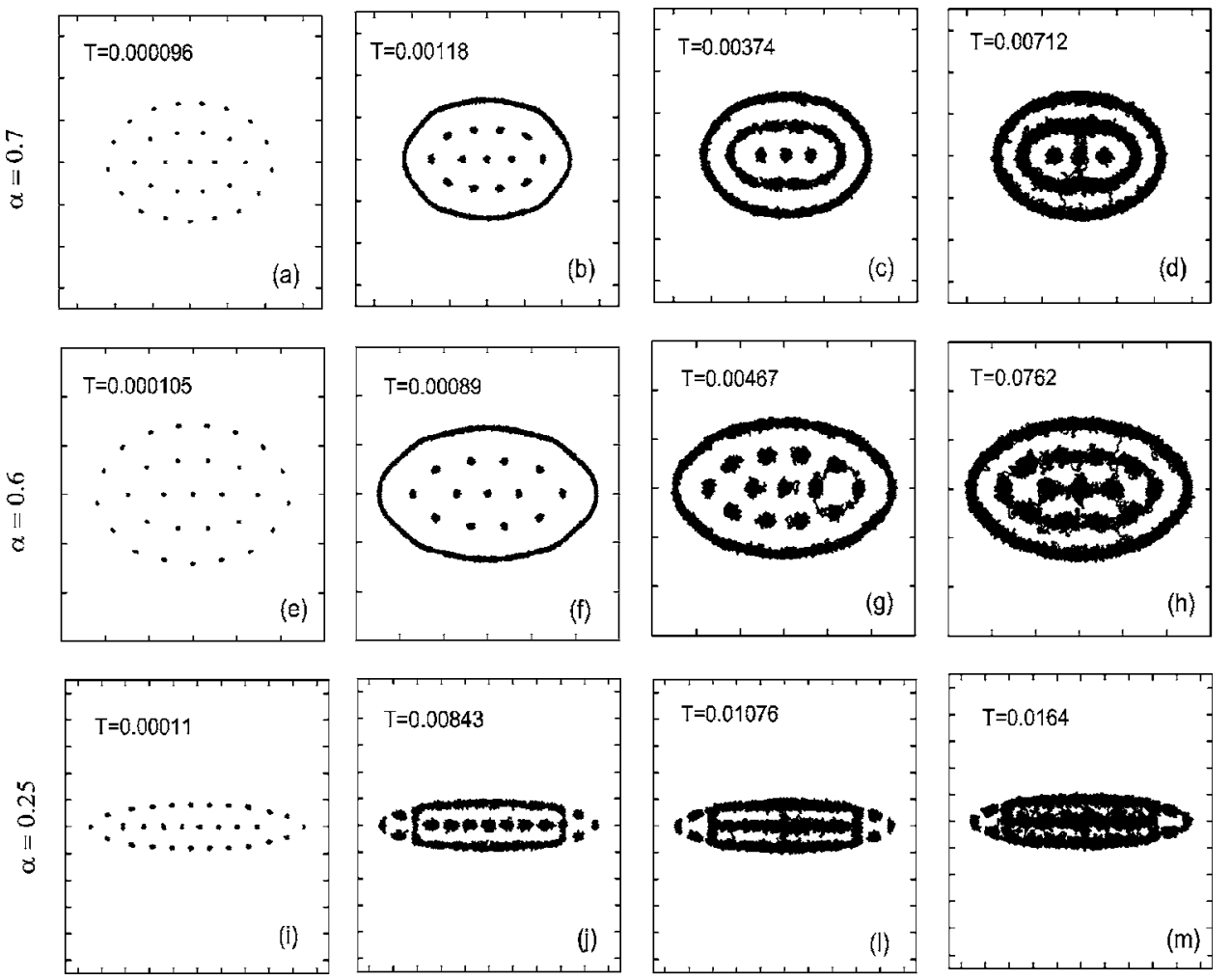

FIG. 6. Particle trajectories obtained for a time interval $\Delta t=500$ for a system with $N=30$ particles interacting through a logarithmic potential and different values of the eccentricity of the confinement potential. The scale is different in each figure, but the distance between the ticks is always a two length unit.
We present in Fig. 6 the trajectory patterns for the system with $N=30$ particles and different values of the eccentricity. For an eccentricity parameter of $\alpha=0.7$ (first row in Fig. 6) and increasing temperature the system first exhibits an intershell melting process at an average temperature of $T=0.00118$ [Fig. 6(b)] where the outer shell can rotate, followed by a second intershell melting process at $T=0.00374$ [Fig. 6(c)] involving the second shell in the cluster. Radial melting occurs at $T=0.00712$ [Fig. 6(d)] where the central particle can jump to the second shell. The arrangement of particles inside the outer shell corresponds to the ground-state configuration for the smaller system with $N=13$ particles and eccentricity $\alpha=0.7$. The melting pattern exhibited by the particles inside the external shell (the first row of Fig. 6) is very similar to the melting pattern exhibited by the system with $N=13$ and $\alpha=0.7$ (the first row of Fig. 3). For an eccentricity of $\alpha=0.6$ (the second row of Fig. 6) an intershell melting process is found at an average temperature of $T=0.00089$ [Fig. 6(f)] for the outer shell. For increasing temperatures we see [Figs. 6(g) and 6(h)] that the system does not exhibit the previous second intershell melting, instead a radial melting takes place for the particles inside the outer shell. The set of particles inside the outer shell of this latter system (the second row of Fig. 6) undergoes a similar melting process like the one found for the system with $N=13$ particles and $\alpha=0.45$ (the third row of Fig. 3). Finally, for a larger anisotropy $\alpha=0.25$, the internal intershell melting process, is now the first melting process, which appears for increasing temperatures [Fig. 6(j)] as found previously for systems with a smaller number of particles, $N=6,12$, and 13
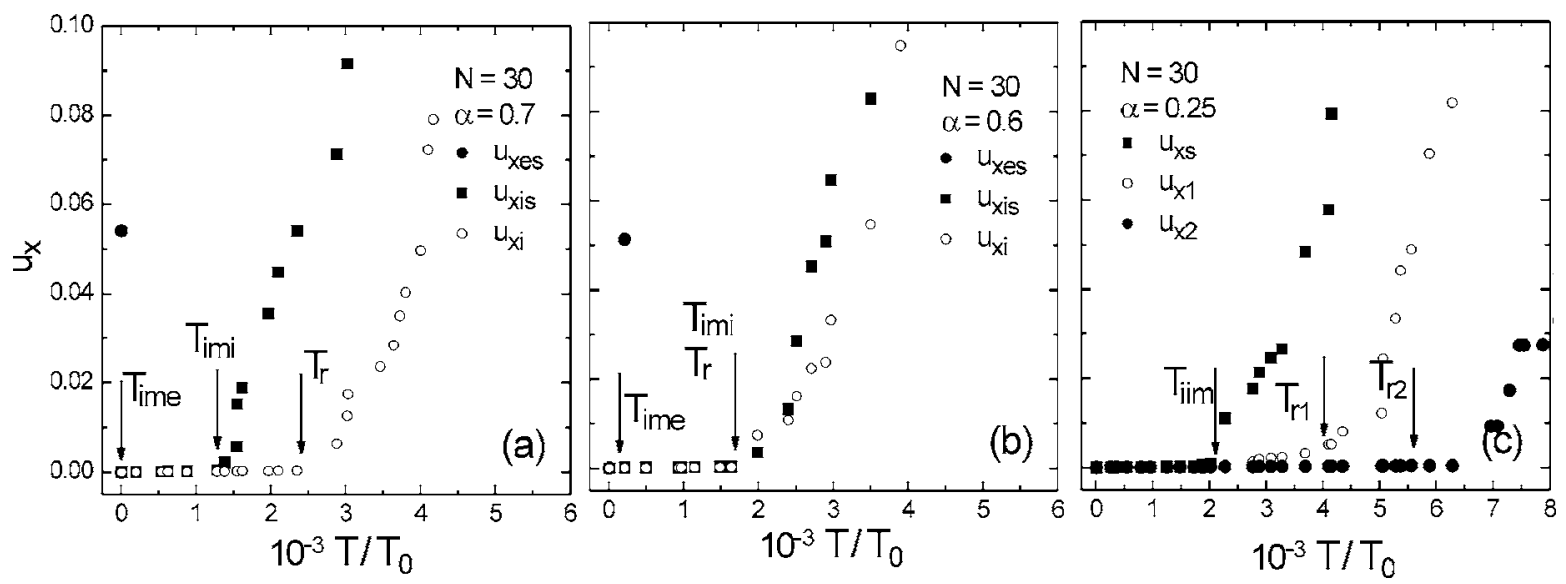

FIG. 7. (a), (b), and (c) show the temperature dependence of the $x$-averaged displacement for the system with $N=30$ particles and the value of the eccentricity confinement of $\alpha=0.7,0.6$, and 0.25 , respectively. In (a) and (b) $u_{x e s}$ increase immediately beyond 0.1 for $T>0$. 


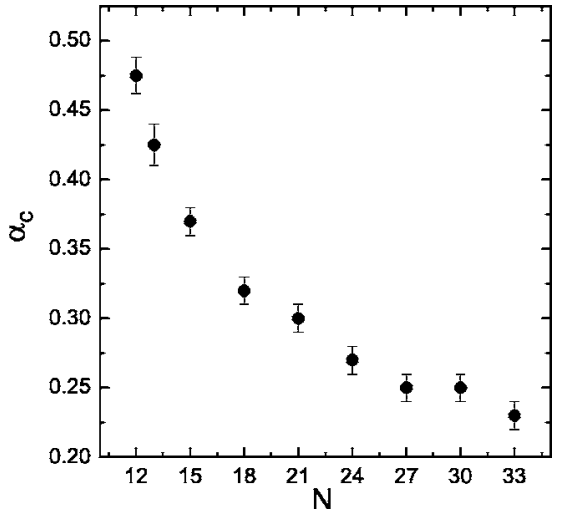

FIG. 8. Maximum values of the anisotropy confinement, as a function of the number of particles, for which internal intershell melting occurs.

particles. We can see in Fig. 6(j) that the three particles in the lateral extremum at both sides of the cluster oscillate around their equilibrium position while a shell involving 17 particles in the center of the cluster is formed. This shell encloses seven particles, which forms a line in the center of the shell and develops an oscillatory motion. For increasing temperature, radial melting occurs [Fig. 6(1)] at a temperature $T$ $=0.01076$ where particles inside the shell can jump to the shell and vice versa. We notice that for this melting stage both groups of three particles beside the shell still do not melt and exhibit an oscillatory motion. For a higher temperature of $T=0.0164[$ Fig. $6(\mathrm{~m})]$ the system exhibits full melting involving all particles in the system.

The temperature dependence of the $x$-averaged displacement and the melting temperature for the systems with $N=30$ particles and $\alpha=0.7,0.6$, and 0.25 are shown in Fig. 7 . For an eccentricity of $\alpha=0.7$, Fig. 7(a) shows the dependence of the $x$-averaged displacement as a function of temperature for the particles inside the external shell and inside the internal shell, $u_{x e s}$ and $u_{x i s}$, respectively, and for the groups of particles enclosed by the internal shell, $u_{x i}$. The sudden increase of these quantities determine, respectively, the following melting temperatures: intershell melting temperatures related to the external and internal shells, $T_{\text {ime }}$ and $T_{\text {imi }}$, respectively, and the radial melting temperature, $T_{r}$. For the latter system, the external and internal shells exhibit an intershell melting process at $T_{\text {ime }}=0.006 \times 10^{-3}$ and $T_{i m i}=1.28 \times 10^{-3}$, respectively, for increasing temperatures. A radial melting process occurs at $T_{r}=2.41 \times 10^{-3}$. For an eccentricity of $\alpha=0.6$, the value of the intershell melting temperature associated with the external shell is $T_{\text {ime }}=0.22$ $\times 10^{-3}$. Now no angular melting related to the internal shell is observed. For this situation both $x$-averaged displacements $u_{x i s}$ and $u_{x i}$ simultaneously diverge at the temperature $T_{r}=1.64 \times 10^{-3}$. Finally, Fig. 7 (c) presents the melting temperature for the system with $\alpha=0.25$. An internal intershell melting occurs at temperature $T_{i i m}=2.02 \times 10^{-3}$. This latter is followed by two radial melting processes: first, a melting process involving the particles in the center of the cluster, which happen at $T_{r 1}=4.15 \times 10^{-3}$ and second, a melting of the group of particles positioned in the edges of the cluster that occurs at the temperature $T_{r 2}=5.56 \times 10^{-3}$.

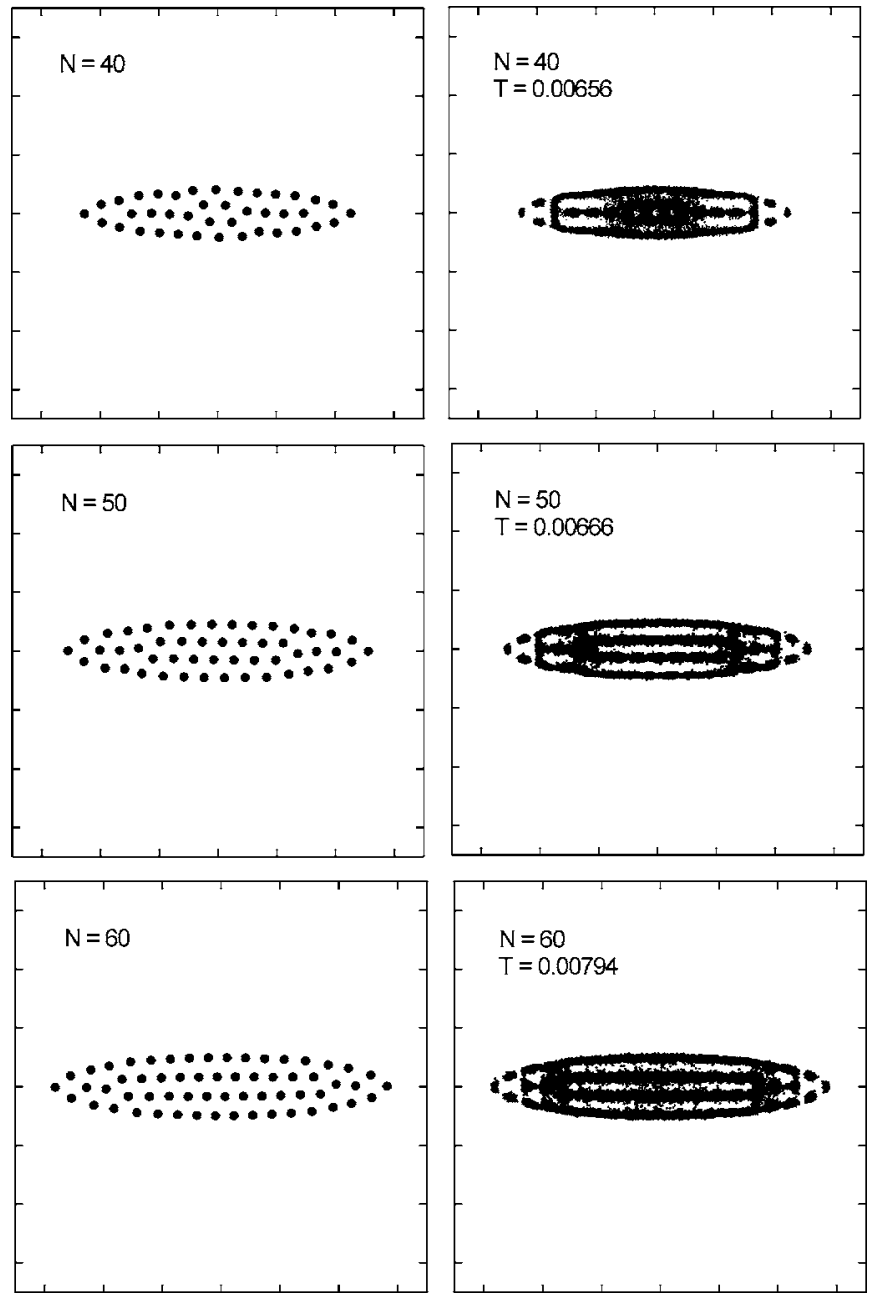

FIG. 9. From top to bottom: the first column shows the groundstate configurations for systems with $\alpha=0.22$ and $N=40,50$, and 60 , respectively. The second column exhibits the temporal trajectory of the same systems at some specific temperature. The distance between the ticks is five length units.

In order to better understand how internal intershell melting depends on the size of the cluster, we calculated the critical eccentricity parameter, defined as the largest eccentricity for which the internal intershell melting occurs, for the following different systems: $N=12,13,15,18,21,24,27,30$, and 33 particles (Fig. 8). Transitions between the different melting phases of small systems is continuous (only in the thermodynamic limit do we have a well-defined phase transition). To calculate the critical eccentricity parameter we first calculated the $x$-averaged displacements $u_{x s}, u_{x 1}$, and $u_{x 2}$ similarly, as was previously done for the system of $N=30$ particles and $\alpha=0.25$ (Fig. 7). Internal intershell melting occurs only if the averages $u_{x s}, u_{x 1}$, and $u_{x 2}$ increase rapidly over a small temperature range. We determined both the highest value of the eccentricity parameter for which the iim occurs and the lowest value of the eccentricity parameter for which iim no longer occurs. The critical eccentricity parameter was then defined as the geometric average of those values and the error bar as half of their difference. From Fig. 8 we notice that the internal intershell melting is always 

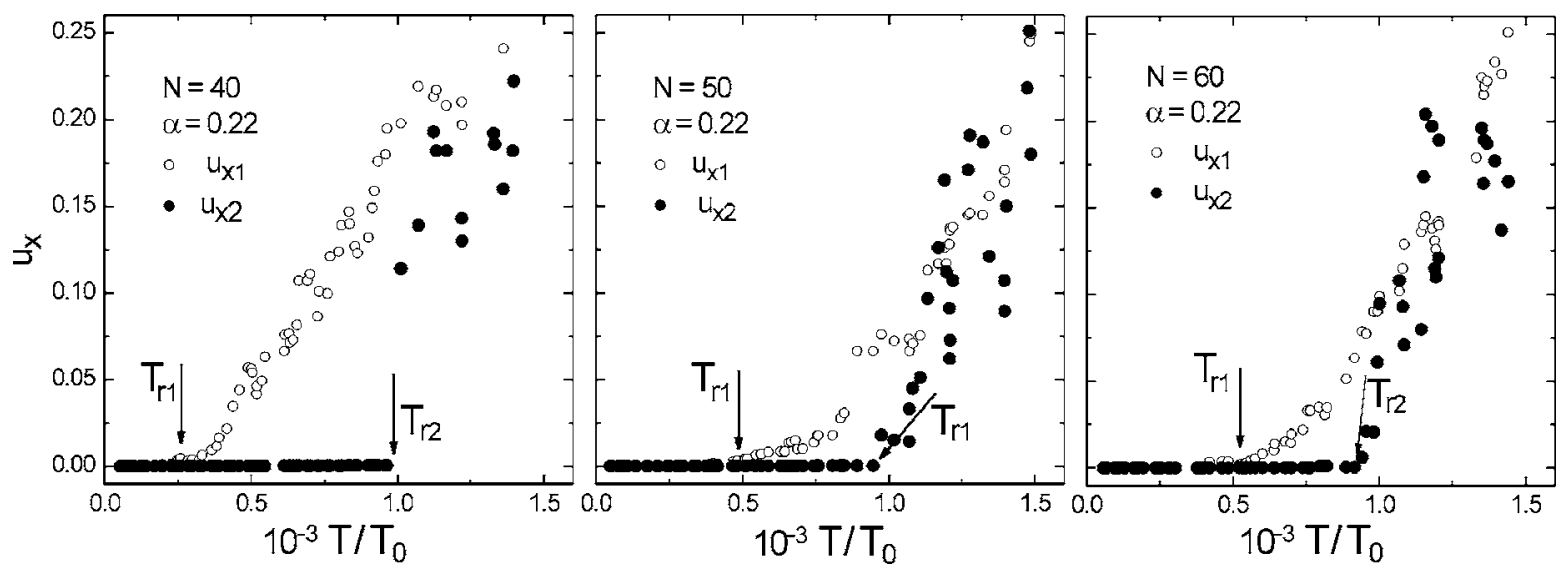

FIG. 10. (a), (b), and (c) show, respectively, the temperature dependence of the $x$-averaged displacement for systems with $N=40,50$, and 60 particles and for a value of the eccentricity confinement of $\alpha=0.22$.

present in small size systems and furthermore, that the value of the critical eccentricity decreases as the number of particles increases. Systems with more than 33 particles were found in an undefined dynamic phase between internal intershell melting and inhomogeneous melting. Additionally, we investigated larger systems such as $N=40,50$, and 60 particles using a qualitative (Fig. 9) and quantitative (Fig. 10) approach. The first and second columns of Fig. 9 show, respectively, the ground-state configurations and the melting patterns for the larger systems. Their melting pattern indicates that the set of three particles in the far left and right of the clusters have a higher melting temperature than the cen- ter region of the cluster. To confirm this quantitatively we calculated the $x$-average displacements $u_{x 2}$ and $u_{x 1}$ (Fig. 10) related, respectively, with the set of three particles placed on the extremum left and right edges of the cluster and the region between these clusters, i.e., the center region. We confirm that inhomogeneous melting pervades for large systems, where the regions at the edges exhibit higher melting temperature contrasting with the dynamic properties found in the rest of the cluster. In other words, $u_{x 1}$ and $u_{x 2}$ increase rapidly for different values of the temperature. We found (Fig. 10) that the melting temperature for clusters with $N=40,50$, and 60 are $T_{r 2}=10.11 \times 10^{-3}, 9.47 \times 10^{-3}$, and $9.54 \times 10^{-3}$,
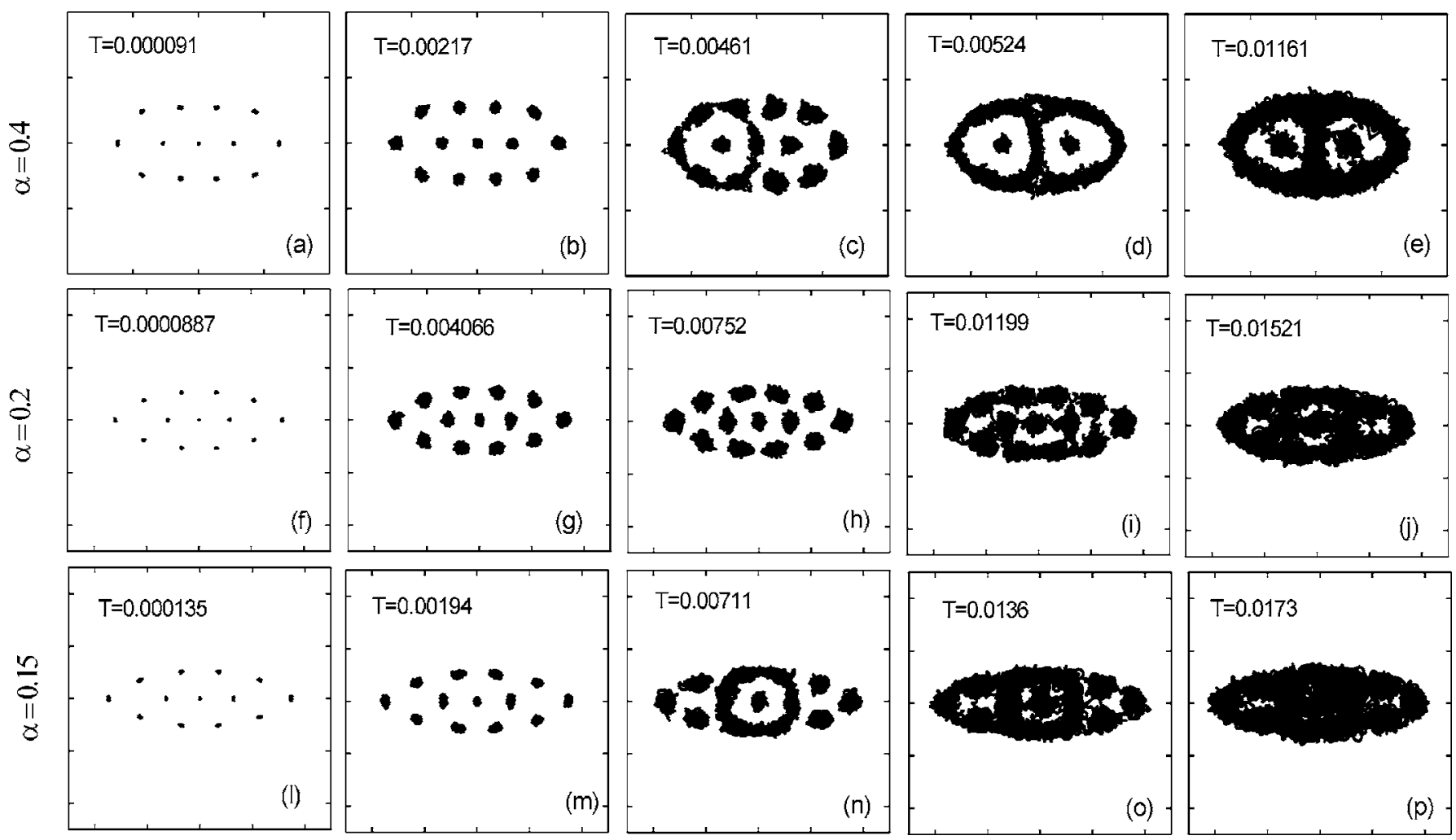

FIG. 11. Particle trajectories obtained during a time interval $\Delta t=500$ for a system with $N=30$ particles. Each row corresponds to the same value of $\alpha$ and along the column from left to right the temperature is increased. The distance between the ticks is one length unit. 


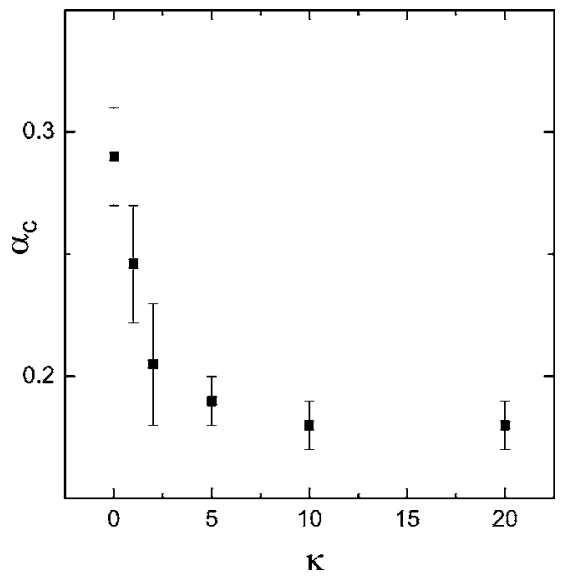

FIG. 12. Maximum values of the anisotropy confinement as a function of the screening parameter for which the internal intershell melting occurs.

respectively. The region in the middle of the clusters has lower melting temperatures: $T_{r 1}=3.22 \times 10^{-3}, 4.82 \times 10^{-3}$, and $5.41 \times 10^{-3}$, respectively.

\section{MELTING TRANSITION FOR SYSTEMS WITH SCREENED COULOMB INTERPARTICLE POTENTIAL}

In the previous section we found that anisotropic confinement strongly influences the melting process of classical particles interacting through a logarithmic interparticle potential. We saw that new melting processes appear, as for example, the internal intershell melting process. Many systems however, for example, dusty plasmas, do not interact through a logarithmic potential but through a screened Coulomb interparticle potential. In this section we investigate the dependence of the new melting processes on the range of the interparticle interaction. First, we show the temporal trajectories for the system with $N=13$ particles and screening parameter $\kappa=5$. The melting process found for this latter system is compared with the one present for the system with logarithmic interparticle potential for which the typical trajectory patterns were shown in Fig. 3. Secondly, we show the relation between the screened Coulomb parameter $\kappa$ and the eccentricity of the confinement potential $\alpha$ for which internal intershell melting can occur.

The trajectory patterns for the system with $N=13$ particles, $\kappa=5$, and different values of the eccentricity of the confinement potential are displayed in Fig. 11. For an anisotropic confinement of $\alpha=0.4$ (the first row of Fig. 11), the first melting process occurs at $T=0.00461$ [Fig. 11(h)] and it involves only the particles in the left side of the cluster. The reason for such asymmetric melting is due to the short time interval over which this trajectory pattern is shown. The time interval was too short for the system to visit the complete accessible phase space. For higher temperature $(T=0.00524)$, both groups of particles in the left and right sides of the cluster participate in the melting process. Figure 11(j) shows that for $T=0.01161$ radial melting has set in. This local intershell melting, which involves particles in the lateral areas of the cluster, is different from the intershell melting found in the previous system with logarithmic interparticle potential whose trajectory pattern is shown in the first and second rows of Fig. 3, respectively, at an anisotropic confinement of $\alpha=0.7$ and $\alpha=0.6$. In the latter case the intershell melting involves all particles in the shell while for local intershell melting, particles in the shell start to form two groups of particles, which are subjected to independent melting processes. The local intershell melting in the system is a consequence of the short-range interaction potential when particles in opposite sides of the shell have their repulsive potential decreased. With increased anisotropy $(\alpha=0.2)$ the radial melting process becomes the first melting process to occur with increasing temperature. This effect of the anisotropic confinement on the melting process was also verified previously for the system with logarithmic interparticle potential (the thirdrow of Fig. 3). The internal intershell melting process is the first melting process for the system with anisotropy $\alpha=0.15$ (the third row of Fig. 11). This phenomenon also occurred for the system with logarithmic interparticle potential but only for an eccentricity of $\alpha=0.4$.

In the absence of screening, i.e., for $\kappa=0$, previous studies $[16,29,30]$ have shown that the particles arrange themselves in rings. The number of particles on each ring and the number of rings depend on $N$ and were cataloged into a Mendeleev-type of table [16,29]. For small values of $\kappa$ we still recover this ring structure; the population of each ring can be a function of $\kappa$, but for sufficiently large screening the particles arrange themselves into a finite-size triangular Wigner crystal. Even in a system with anisotropic confinement, increasing the screening parameter is able to induce a triangular arrangement of particles. We found that the internal intershell melting also appears for systems with a value of the screening parameter of $\kappa=0,1,2,10$, and 20 . We found that for each of these screening parameters there is a maximum value of the eccentricity parameter $\alpha$ of the confinement for which the internal intershell melting is able to occur, which we call the critical eccentricity $\alpha_{c}$. Figure 12 shows that the value of the critical eccentricity $\alpha_{c}$ decreases with increasing screening parameter $\kappa$. This is a consequence of the formation of a triangular arrangement of the particles with increasing screening parameter. Increasing the anisotropic confinement further recovers the arrangement of Fig. 3(q) and internal intershell melting becomes possible again.

\section{SUMMARY AND CONCLUSIONS}

The effect of the eccentricity of the confinement potential on the melting process of anisotropically confined particles interacting through a repulsive interparticle potential was investigated. First, a qualitative investigation concerning shorttime temporal trajectories performed for small and intermediate size systems with $N=6,12,13$, and 30 particles and different values of the eccentricity confinement revealed the principal melting properties of anisotropic systems. Angular oscillation substantially influences the dynamic processes in weakly anisotropic confined clusters. At first sight, increasing the anisotropy of the confinement diminishes the importance of angular oscillation on the dynamic of the system. However, for some critical value of the eccentricity, the an- 
gular melting process is recovered through a pattern formation called internal intershell melting. This latter dynamic mechanism can be well understood in terms of the moment transferred between particles belonging to the same shell.

We found that increasing the anisotropy of the confinement potential drives the system through the following different melting sequences: (1) a similar melting pattern as present in a circular system, i.e., intershell melting followed by radial melting; (2) there is only radial melting, and (3) an angularlike melting appears, which we call internal intershell melting since it involves only the particles located in the central region of the cluster. This latter phase transition is followed by radial melting.

The influence of the number of particles on the melting process is as follows. For small systems, for example, $N=12$ and 13 particles both with eccentricity $\alpha=0.4$, the arrangement of the particles strongly influences the value of the internal intershell melting temperature which reminds us to the dynamic stability found in magic-number configurations for isotropically confined systems. This is a direct con- sequence of the triangular arrangement on the angular melting process. Furthermore, we found that for larger systems $(N=40,50$, and 60 were considered) internal intershell melting is not present anymore but inhomogeneous melting still persists.

We confirmed that inhomogeneous melting is present independently of the range of the interparticle interaction by considering Coulomb and screened Coulomb potentials and should therefore be observed in systems as dust plasmas, colloidal suspensions, and metallic balls under anisotropic confinement. In summary, the main message of the paper is inhomogeneous melting occurs in anisotropically confined clusters and its appearance in small systems occurs in a distinct form called here internal intershell melting.

\section{ACKNOWLEDGMENTS}

This work was supported by the Flemish Science Foundation (FWO-Vl). We thank M. Saint Jean for discussions.
[1] C. C. Grimes and G. Adams, Phys. Rev. Lett. 42, 795 (1979).

[2] R. Ashoori, Nature (London) 379, 413 (1996).

[3] M. Golosovsky, Y. Saado, and D. Davidov, Phys. Rev. E 65, 061405 (2002).

[4] G. Blatter, M. V. Feigel'man, V. B. Geshkenbein, A. I. Larkin, and V. M. Vinokur, Rev. Mod. Phys. 66, 1125 (1994).

[5] A. K. Geim, I. V. Grigorieva, S. V. Dubonos, J. G. S. Lok, J. C. Maan, A. E. Filippov, and F. M. Peeters, Nature (London) 390, 259 (1997).

[6] J. H. Chu and Lin I, Phys. Rev. Lett. 72, 4009 (1994).

[7] M. Saint Jean, C. Even, and C. Guthmann, Europhys. Lett. 55, 45 (2001).

[8] M. Saint Jean and C. Guthmann, J. Phys.: Condens. Matter 14, 13653 (2002).

[9] G. B. Hess, Phys. Rev. 161, 189 (1967).

[10] Y. Pearl, Appl. Phys. Lett. 5, 65 (1964).

[11] A. I. Buzdin and J. P. Brison, Phys. Lett. A 196, 267 (1994).

[12] L. R. E. Cabral and F. M. Peeters, Phys. Rev. B 70, 214522 (2004).

[13] E. P. Wigner, Phys. Rev. 46, 1002 (1934).

[14] R. S. Crandall and R. Williams, Phys. Lett. 34A, 404 (1971).

[15] L. Bonsall and A. A. Maradudin, Phys. Rev. B 15, 1959 (1977).

[16] V. M. Bedanov and F. M. Peeters, Phys. Rev. B 49, 2667
(1994).

[17] S. W. S. Apolinario, B. Partoens, and F. M. Peeters, Phys. Rev. E 72, 046122 (2005).

[18] L. Cândido, J. P. Rino, N. Studart, and F. M. Peeters, J. Phys.: Condens. Matter 10, 11627 (1998).

[19] A. P. Young, Phys. Rev. B 19, 1855 (1979).

[20] D. R. Nelson and B. I. Halperin, Phys. Rev. B 19, 2457 (1979).

[21] J. M. Kosterlitz and D. J. Thouless, J. Phys. C 6, 1181 (1973).

[22] D. R. Nelson and B. I. Halperin, Phys. Rev. B 19, 2457 (1979).

[23] A. H. Marcus and S. A. Rice, Phys. Rev. E 55, 637 (1997).

[24] K. J. Strandburg, Rev. Mod. Phys. 60, 161 (1988).

[25] F. M. Peeters, V. A. Schweigert, and V. M. Bedanov, Phys. Plasmas 212, 237 (1995).

[26] V. A. Schweigert and F. M. Peeters, Phys. Rev. B 51, 7700 (1995).

[27] Yu. E. Lozovik and V. A. Mandelshtam, Phys. Lett. A 145, 269 (1990).

[28] L. Verlet, Phys. Rev. 159, 98 (1967); 165, 201 (1967).

[29] B. Partoens and F. M. Peeters, J. Phys.: Condens. Matter 9, 5383 (1997).

[30] F. Bolton and U. Rössler, Superlattices Microstruct. 13, 139 (1993). 